\title{
Infraestructura tecnológica para supermercados
}

\author{
Technological infrastructure for supermarkets
}

\author{
Infraestrutura tecnológica para supermercados
}

\author{
Sergio Andrés Rossells Lovera \\ sergiorossells@gmail.com \\ ORCID 0000-0003-1665-4183
}

Universidad Privada del Valle, Cochabamba-Bolivia

Artículo recibido 8 septiembre 2020 | Arbitrado del 3 al 27 octubre 2020 | Publicado el 1 enero 2021

RESUMEN

En el ámbito empresarial, es cada vez más necesario implementar arquitectura de seguridad electrónica e infraestructura de telecomunicaciones, la complejidad en el diseño de estos sistemas exige aplicar un proceso sistemático específico para el rubro en el que la empresa se desenvuelve. En este sentido, el objetivo de este estudio fue proponer un proceso para el dimensionamiento de soluciones de infraestructura tecnológica integral, específica para supermercados. La investigación se desarrolló en dos fases: una fase documental que, mediante el método bibliográfico y consultas a expertos, permitió crear el proceso propuesto y una fase de campo que permitió su validación en un supermercado ubicado en La Paz, Bolivia. Como resultado se cuenta con un proceso iterativo capaz de integrar sistemas de redes de datos, seguridad, CCTV, control de acceso y cableado estructurado. La aplicación del proceso generó ahorro en tiempo, recurso humano y tecnología. El proceso podrá aplicarse en otros contextos.

Palabras clave: Red de datos; seguridad electrónica; CCTV; control de acceso; cableado estructurado; infraestructura tecnológica
ABSTRACT

RESUMO
In the business field, it is increasingly necessary to implement electronic security architecture and telecommunications infrastructure, the complexity in the design of these systems requires applying a specific systematic process for the area in which the company operates. In this sense, the objective of this study was to propose a process for the dimensioning of comprehensive technological infrastructure solutions, specific for supermarkets. The research was developed in two phases: a documentary phase that, through the bibliographic method and consultations with experts, allowed to create the proposed process and a field phase that allowed its validation in a supermarket located in La Paz, Bolivia. As a result, there is an iterative process capable of integrating data network systems, security, CCTV, access control and structured cabling. The application of the process generated savings in time, human resources and technology. The process may be applied in other contexts.

Key words: Data network; electronic security; CCTV; access control; structured cabling; technological infrastructure
No campo empresarial, é cada vez mais necessária a implementação de arquitetura de segurança eletrônica e infraestrutura de telecomunicações, a complexidade no projeto desses sistemas exige a aplicação de um processo sistemático específico para a área em que a empresa atua. Nesse sentido, o objetivo deste estudo foi propor um processo de dimensionamento de soluções integrais de infraestrutura tecnológica, específicas para supermercados. A pesquisa foi desenvolvida em duas fases: uma fase documental que, por meio do método bibliográfico e consultas a especialistas, permitiu a construção do processo proposto e uma fase de campo que permitiu sua validação em um supermercado localizado em La Paz, Bolívia. Como resultado, existe um processo iterativo capaz de integrar sistemas de redes de dados, segurança, CFTV, controle de acesso e cabeamento estruturado. A aplicação do processo gerou economia de tempo, recursos humanos e tecnologia. 0 processo pode ser aplicado em outros contextos.

Palavras-chave: Rede de dados; segurança eletrônica; CCTV; controle de acesso; cabeamento estruturado; infraestrutura tecnológica 
INTRODUCCIÓN

Los avances tecnológicos generan oportunidades diversas de crecimiento en el ámbito empresarial, la implementación y capacidad de gestión de estos avances proporcionará una ventaja competitiva para todo tipo de organización (Cano-Pita. 2018). Esta relación no es menor si analizamos concretamente la industria de la venta de alimentos al por menor, la tecnología es una variable que mueve la competitividad en cadenas de supermercados (Dallabona, 2019), la tecnologización es necesaria.

Si bien es necesario que la organización avance en paralelo a la evolución de los cambios tecnológicos, sin embargo, este desarrollo trae consigo nuevas necesidades de protección y seguridad. Los recursos que incorporan tecnología deben ser protegidos de accesos, tanto internos como externos, no autorizados a través de recursos de software y hardware para garantizar la autenticidad, confidencialidad e integridad de la información (Migga, J. 2017). La seguridad electrónica e infraestructura de telecomunicaciones debe acompañar los procesos de innovación en todos los sectores.

Los ataques realizados para robar información corporativa se han incrementado considerablemente (Malware, suplantación de identidad, ataques del hombre en el medio, Inyecciones SQL, ataques a contraseña, Rootkits y secuencia de comandos entre otros), comprometiendo todo tipo de empresas, desde pequeñas hasta multinacionales, creando la necesidad de agregar dispositivos de seguridad a la red a prueba de ataques cibernéticos cada día más complejos.

Los supermercados, y otros sectores que se caracterizan por tener alto tráfico de clientes en sus instalaciones, además se ven afectados por otro factor que acompaña el desarrollo tecnológico: el creciente uso de dispositivos móviles, aplicaciones en línea y redes sociales, que acrecienta el tráfico irrelevante en empresas, sobrecargando las conexiones a internet y reduciendo el ancho de banda destinado a aplicaciones críticas para el funcionamiento de la institución. Entonces surge la necesidad de realizar control sobre el tráfico.

Dimensionar, diseñar e implementar arquitecturas de seguridad electrónica e infraestructura de telecomunicaciones implica contar con especialistas en diferentes áreas que además de considerar la teoría, normativa, especificaciones de fabricantes, cuenten con una vasta experiencia práctica. La ocurrencia de errores en estos procesos significa consumir innecesariamente recursos monetarios, humanos y temporales. En este sentido, este estudio propone un proceso para el dimensionamiento de soluciones de infraestructura tecnológica integral, específico para supermercados.

No se ha encontrado en la literatura una metodología o proceso que marque las directrices para el diseño de soluciones integrales de infraestructura tecnológica, se entiendo que tener un mecanismo para la integración de los sistemas permitirá asegurar que se tomen en cuenta todas las variables para proponer el diseño más costo eficiente. El buen diseño de componentes va a simplificar la tarea de adquisición de equipos, definición de alcances y manejo de expectativas.

Si bien Bolivia es un país en vías de desarrollo, la implementación de sistemas tecnológicos es inminente, cada vez es más frecuente que las organizaciones demanden soluciones de infraestructura tecnológica. Entonces, las empresas del rubro deben 
responder con eficiencia. Aquí se materializa la pertinencia del estudio propuesto, ya que provee de una herramienta que coadyuva a la consecución de ventajas competitivas para los proveedores de infraestructura tecnológica.

\section{METODOLOGÍA}

Se trata de una investigación desarrollada en dos fases, documental y de campo. En una primera fase se visualiza el componente documental que consistió en recoger el conocimiento teórico y empírico disponible en fuentes secundarias $\mathrm{y}$ terciarias, relacionado con diseño, dimensionamiento e implementación de soluciones tecnológicas. Asimismo, gracias a la consulta a expertos, mediante entrevistas semiestructuradas, se recogió también el conocimiento de especialistas en estas áreas. El análisis y síntesis de la información recogida mediante estos tres tipos de fuentes de información permitió proponer un proceso iterativo para el dimensionamiento de soluciones de infraestructura tecnológica integral, específico para supermercados.

A continuación, durante la segunda fase del estudio, se trató de una investigación de campo porque el proceso propuesto fue aplicado en una agencia supermercado de la ciudad de La Paz, Bolivia. Esta fase, de corte transversal, consistió en representar el proceso de diseño de la infraestructura que soporta: red de datos, seguridad de red, CCTV, control de acceso, energía ininterrumpida y cableado estructurado de red para una sucursal nueva de supermercado. Para esto, se contó con un equipo multidisciplinario acreditado, conformado por tres ingenieros en redes, servidores y seguridad.
Dado que tanto la creación como la aplicación del proceso propuesto implicaron contar con diferentes especialistas, se usaron diversos métodos y herramientas que se explican a continuación.

En cuanto a los métodos, inicialmente se aplicó el método bibliográfico para recoger información técnica de todas las áreas que incluye el proceso, así: a) Para las tareas de redes de datos se consideraron las pautas propuestas en las guías de Allred (2018) y Allied Telesis (2020), complementando con los manuales de los fabricantes. Durante la elaboración del diseño de cada componente se tuvo el apoyo directo de los fabricantes de equipos de red. b) Por su parte, en las tareas de CCTV y control de acceso se consideraron las pautas propuestas por Sennewald (2012). Asimismo, se realizó una revisión de las pautas propuestas por los fabricantes HIKvision y la reglamentación existente en Bolivia, según Resolución Ministerial No. 153/15 (2015), acerca de videovigilancia en lugares de trabajo. c) Las tareas de diseño del cableado estructurado consideraron las pautas propuestas por Cadenas, Zaballos y Salas (2012) y las normas de las instalaciones de cableado estructurado de ANSI/TIA/EIA-568, ANSI/TIA/EIA-569, ANSI/TIA/EIA-606 y ANSI/TIA/EIA-607.

A continuación, se aplicó el método de análisis y síntesis. Dado que ya se contaba con toda la orientación técnica plasmada en la literatura, se procedió a consultar la percepción de especialistas con experiencia en dimensionamiento de soluciones de infraestructura tecnológica integral en supermercados y centros comerciales. Esto se realizó mediante entrevistas semiestructuradas y grupos de trabajo. Es así como gracias al conocimiento teórico y práctico se pudo sinterizar y proponer las 
etapas y condiciones que debe cumplir el proceso.

En la investigación de campo, segunda fase de esta investigación, se aplicaron las siguientes herramientas de cálculo y dimensionamiento: tablas de cálculo (Excel), herramientas de dibujo asistido (AutoCAD, Visio) y herramientas propias para este propósito.

Los resultados que se presentan a continuación siguen estas dos fases: Primero se expone el proceso propuesto, se describe la importancia y modo de aplicar de cada una de sus 4 etapas, describiendo a su vez las condiciones requeridas para su aplicación. Segundo, se presenta un caso práctico de su aplicación en un supermercado. Esta etapa es bastante detallada, describe poco a poco como se desarrolló cada una de las 4 etapas y, de la manera más explícita posible, se explica las condiciones para su aplicación (según pide el proceso propuesto).

\section{RESULTADOS}

Para garantizar el correcto dimensionamiento de la infraestructura tecnológica se propone un proceso iterativo capaz de integrar 5 sistemas: redes de datos, seguridad, CCTV, control de acceso y cableado estructurado. El proceso requiere la aplicación de cuatro etapas (generalidades, relevamiento de variables, diseño de arquitectura, consenso) descritas en el gráfico 1.

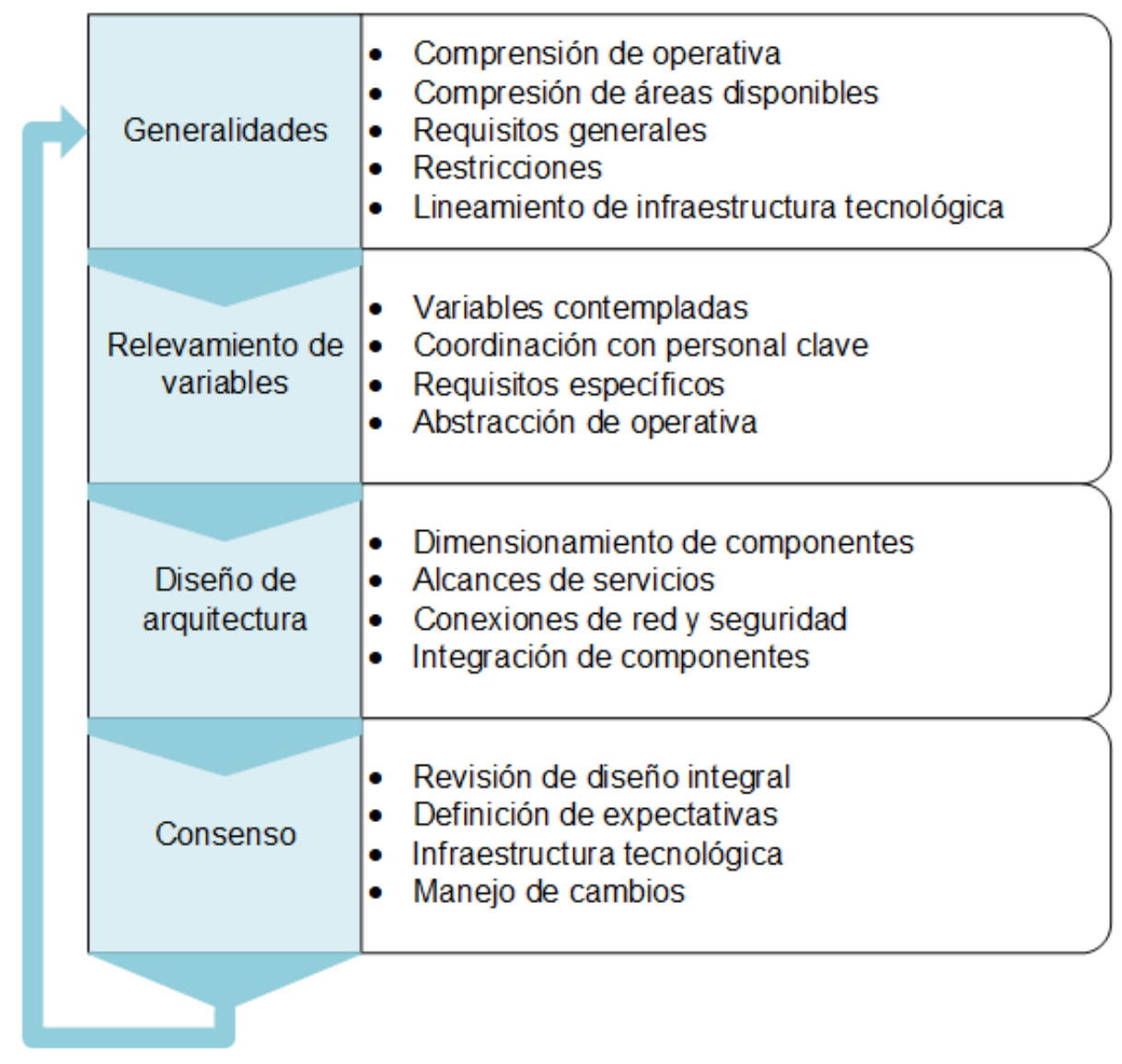

Gráfico 1. Etapas del proceso de dimensionamiento. 
El gráfico 1 muestra en resumen las cuatro etapas del proceso propuesto y la información relevante que se procura en cada etapa. Este proceso puede iterar para realizar ajustes de presupuesto y cambios en el requerimiento original. Para comprender a cabalidad este proceso, primero se describirá cada etapa desglosando a su vez las condiciones requeridas para su aplicación.

\section{Generalidades}

Esta etapa inicia por la comprensión de la operativa de negocio, implica responder a una serie de preguntas: ¿Qué se va a hacer? ¿Qué se va a comercializar? ¿Existen servicios complementarios? ¿Cuáles son los servicios asociados? ¿Se trata de una agencia central o una sucursal? ¿Cuál es el presupuesto disponible?

Una vez se conoce esta información, se procede al reconocimiento de la infraestructura del lugar, se obtienen los planos a escala, línea y nivel, áreas disponibles para equipamiento, losas, entretechos y pisos técnicos.

En esta temprana etapa, se debe tener en cuenta qué sistemas se van a interconectar, para establecer los requisitos generales relacionados con cada uno de estos sistemas. Asimismo, se deberán registrar las restricciones temporales, presupuestarias y legales.

Los lineamientos de infraestructura tecnológica están en función a preferencias de fabricantes $\mathrm{y} / \mathrm{o}$ tecnologías propietarias, disponibilidad y/o integración a un sitio de contingencia, servicios en nube, etc. Estos lineamientos también dependen de si se trata de una infraestructura nueva, una renovación o crecimiento.

A lo largo de esta primera etapa es necesario mantener comunicación directa con el representante del negocio, personal técnico y ejecutivos.

\section{Relevamiento de variables}

Con el input obtenido en la etapa 1, es posible realizar un relevamiento de variables independiente para cada sistema. Sin embrago, debe considerarse que las variables de un sistema pueden ser entradas para el siguiente. En términos generales se sugiere empezar por el equipamiento de acceso.

De manera similar a la etapa anterior, es importante definir el personal clave con quien se coordinará. En el caso de un supermercado, el encargado de tecnología es quien da el lineamiento tecnológico, por su parte el encargado de sala de ventas va a dar pautas de la operativa de negocio, finalmente, el encargado de compras y el administrador dan las restricciones de presupuesto. En el marco de esta coordinación, se procede a definir los requisitos específicos para cada una de las variables del sistema. En cada iteración se van puliendo los requisitos hasta llegar a definir completamente las facultades de cada sistema.

Una vez definidos los requisitos específicos, se precisa el personal que estará a cargo y con acceso para administrar los sistemas, permitiendo definir responsabilidades y requerimientos de capacitaciones.

\section{Diseño de arquitectura}

Una vez se cuenta con toda la información recolectada en el relevamiento de variables y las generalidades del caso, se inicia con el dimensionamiento de componentes de cada sistema.

Para cada sistema se deben establecer los alcances de servicios, esto delimita los 
servicios de instalación y entrega del sistema en operación a los encargados de administración. Asimismo, define el nivel de soporte y los niveles de servicio bajo los acuerdos que los usuarios requieran.

Se identifican las conexiones de red entre componentes de cada sistema y se deben contemplar los requerimientos de seguridad según el lineamiento de la empresa y bajo las mejores prácticas de los fabricantes.

Se debe considerar el orden de los sistemas a diseñar para facilitar la consolidación de información. Se procede con el diseño de cada sistema iniciando por los sistemas que tienen componentes más al borde de la red (ej. usuarios, cámaras, dispositivos), cada uno de estos presentara un puerto de red a la capa de acceso de red. Posteriormente, todos los switches de acceso deberán concentrarse en un core de red. A su vez, el diseño de red producirá la información para el diseño del cableado estructurado. El diseño integral de los sistemas observados garantizará la correcta provisión de equipamiento.

\section{Consenso}

Gracias a cada iteración, finalmente, el diseño producido se acercará más a satisfacer las necesidades de la empresa ajustándose para cumplimiento de restricciones, consideraciones y cambios nuevos. Cada revisión del diseño podrá cambiar uno o más de los sistemas integrados, el manejo de la información en medios fácilmente manipulables facilitará las tareas para cada versión.

El diseño de componentes con alcances de servicios definidos debe ser presentado al equipo evaluador de la solución. Durante la presentación las expectativas deben ser asentadas para los usuarios y administradores. La delimitación de servicios es crucial para el buen entendimiento entre partes.

Con el diseño completo, se pueden ir definiendo de antemano la nomenclatura, configuraciones y comportamientos de los sistemas. Estas pautas ayudarán a definir las bases para la implementación de la infraestructura tecnológica.

Como último punto, se debe manejar un control de cambios en cada iteración para trazabilidad entre versiones y posibles reajustes hacia versiones anteriores.

\section{Aplicación del proceso en una cadena de supermercados}

El proceso propuesto se aplicó en una cadena de supermercados que desarrolla actividades en La Paz, Bolivia. Específicamente se tomó como objeto de estudio una sucursal que cuenta con una superficie construida de $6300 \mathrm{~m} 2$ distribuidos en dos plantas (el salón de ventas comprende $4480 \mathrm{~m} 2$ ). La sucursal cuenta con 50 góndolas de productos, área de fiambres, carnicería, bodega de bebidas, productos congelados, ropa, equipos eléctricos de hogar y productos varios. Como equipamiento cuenta con áreas destinadas para oficinas con capacidad para 95 personas, sala de capacitación, salas de reuniones, sala de servidores, 12 cajas de atención, panadería, preparación de comida y almacenes. Adicionalmente, una plaza comercial con 14 locales comerciales e islas de productos y servicios. Se realizaron 7 iteraciones del proceso para llegar al diseño final, cada iteración con una menor duración que la anterior. Este documento presenta el diseño final. 


\section{Generalidades}

Esta etapa fue estratégica, dado que permitió conocer las generalidades que delimitaron el diseño propuesto, estas circunspecciones tuvieron relación con la operativa, espacio disponible, requisitos, restricciones y lineamientos. A continuación, se describen su aplicación, siguiendo las condiciones específicas del proceso propuesto.

Compresión de la infraestructura. En relación con la operativa del supermercado, se realizaban operaciones de renovación de la infraestructura del edificio, remodelación de áreas existentes y construcción de oficinas nuevas. Se consideró que la infraestructura pertenencia a un predio mayor con secciones deportivas, banca, comercio y otros servicios.

Compresión del área disponible. La construcción dispuso de dos espacios para equipamiento de tecnología, ambos en planta alta, una sala de servidores al norte y un cuarto de comunicaciones en el lado sur. Se contó con un cielo con andamios para el equipamiento.

Requisitos generales. Se requirió la instalación de la infraestructura tecnológica base de redes para el inicio de operaciones del supermercado. El proyecto contempló el equipamiento de redes, seguridad de redes, CCTV, acceso y cableado estructurado. La conectividad por redes inalámbricas quedó fuera del alcance de este proyecto, se requirió de servicios de instalación y soporte para todos los componentes.

Restricciones. El diseño fue sujeto a restricciones presupuestarias y temporales. Las condiciones temporales implicaron un plazo de entrega ajustado de 70 días para la importación, instalación, configuración y puesta en marcha de equipos activos. El diseño final fue sujeto a un proceso de licitación. Se apuntó a la inauguración y apertura antes de un feriado nacional.

Lineamientos de la infraestructura tecnológica. El proyecto requirió el diseño y selección de los componentes para una infraestructura nueva, presentando una ventaja para el diseño al ser un proyecto greenfield.

\section{Relevamiento de variables}

El relevamiento de las variables de cada sistema fue considerado bajo los requerimientos del supermercado. Las variables de los subsistemas CCTV, control de acceso y asistencia, impactaron directamente en el dimensionamiento de los subsistemas redes de datos y cableado estructurado. Asimismo, las variables de todos los sistemas fueron consideradas integralmente para la producción del diseño completo.

El orden de la definición de variables es importante, ya que cada subsistema de variables alimenta al dimensionamiento del siguiente subsistema. Esta consideración integral cumple el propósito de dimensionar integralmente y no incurrir en sobredimensionamiento de equipamiento de redes, o peor la falta de disponibilidad de recursos para el funcionamiento. El último caso puede representar gastos significativos para ser resuelto.

En esta etapa cada categoría contempla las siguientes consideraciones específicas: variables contempladas, coordinación con personas clave, requisitos específicos $\mathrm{y}$ abstracción operativa. El relevamiento de estas está detallado en su última iteración.

\section{CCTV}

Variables contempladas. Se tomaron en cuenta las áreas de interés, ingresos y salidas, pasillos, patios de maniobra, 
accesos vehiculares y parqueos, para la definición de la cantidad y tipo de cámaras requeridas en el diseño del sistema.

Se tomaron en cuenta criterios de detección, observación, reconocimiento e identificación (DORI) para el dimensionamiento en la resolución de las cámaras. Asimismo, se consideraron también las capacidades de analíticas embebidas en las cámaras como cruce de línea, intrusión, entrada/salida de área. Para reducción de transmisión y almacenamiento de datos se consideraron todas las cámaras para grabación con codificación h.265 (formato vigente a la fecha del estudio). También se tomó en cuenta la sala de monitoreo para el dimensionamiento de acuerdo a la cantidad de cámaras y propósito.

Coordinación con personal clave. A medida que el diseño alcanzó mayor madurez se involucró al personal a cargo del área comercial y disposición de productos en sala.

Requisitos específicos. El sistema CCTV debe ser IP con alimentación PoE.

Abstracción operativa. En esta etapa se toma en cuenta las responsabilidades de manejo de cada subsistema para la capacitación posterior.

\section{Acceso}

Variables contempladas. El control de acceso requiere proteger y controlar el acceso para zonas restringidas en los predios. Oficinas norte y sur, gerencias, almacenes, archivo, tecnología, atención al cliente, racks norte y sur.

Coordinación con personal clave. Se involucró al personal de seguridad en el relevamiento.

Requisitos específicos. Se decidió que el acceso sea con credenciales de proximidad para el ingreso y botón para la salida. Asimismo, se relevó la posición, material y tipo de puerta para producir la lista de materiales de acuerdo con la información. De igual manera, se reveló la cantidad de credenciales requeridas. Las credenciales pueden ser nombradas 0 asignadas temporalmente (visitas, mantenimiento u otros roles).

Abstracción operativa. En esta etapa se tomó en cuenta las responsabilidades de manejo de cada subsistema para la capacitación posterior.

\section{Asistencia}

Variables contempladas. Se determinó que el control de asistencia requiere registrar a los empleados en el ingreso, salida, descanso y/o cambios de turno.

Coordinación con personal clave. Solamente se involucró al personal de tecnología.

Requisitos específicos. Se determinó que: el control de asistencia compartirá los mismos credenciales y registros que el control de acceso, las credenciales serán nombradas, la información producida por el sistema debe ser exportable para procesamiento en otros sistemas.

Abstracción operativa. En esta etapa se toma en cuenta las responsabilidades de manejo de cada subsistema para la capacitación posterior. Este sistema está a cargo de recursos humanos.

\section{Redes de datos}

Variables contempladas. Este segmento consolida los puntos de red requeridos por CCTV, acceso y asistencia en adición a los puntos de red requeridos para usuarios y dispositivos del supermercado. 
Coordinación con personal clave. Para la definición de puntos de red para equipamiento de sala de ventas se revisaron los requerimientos con el jefe de sala.

Requisitos específicos. Se estableció la necesidad de un punto de red por escritorio para usuarios, puntos dobles en salas, puntos triples para cajas y puntos en sala para balanzas electrónicas. La velocidad requerida para acceso es de $1 \mathrm{Gbps}$. El backbone de red debe contemplar mínimamente la misma velocidad. Se requieren puertos de red en la sala de servidores para servidores, almacenamiento, grabadores y UPS. Se requiere considerar holgura para crecimiento.

Abstracción operativa. Los recursos para administración de este segmento fueron enteramente del área de tecnología.

\section{Seguridad de redes}

Variables contempladas. Se consideró la cantidad y tipo de usuarios que van a acceder a las redes internas y externas del supermercado. Asimismo, redes privadas virtuales y accesos directos.

Coordinación con personal clave. Tecnología solamente.

Requisitos específicos. Como seguridad de redes, el supermercado requiere el filtrado de redes entre cajas, servidores, CCTV y otras redes. Todas ellas segmentadas por física o virtualmente por propósito. Adicionalmente, se requiere el filtrado web para las conexiones salientes de usuarios.

Abstracción operativa. Los recursos para administración de este segmento fueron enteramente del área de tecnología.

\section{Cableado estructurado}

Variables contempladas. Se realiza el relevamiento de espacios disponibles y rutas para el tendido de cableado de red. Se realizan visitas en la obra para la verificación de posibles rutas de cableado troncal y de los puntos de acceso para dispositivos.

Coordinación con personal clave. Se sostuvieron reuniones con el personal de diseño arquitectónico y obras civiles para coordinar los tiempos de ejecución de obra y de instalación de equipamiento.

Requisitos específicos. Para obtener una infraestructura estándar y futurible se registró la necesidad de cableado de red CAT6 mínimamente para el tendido de cobre y OM4 para el tendido de fibra. Asimismo, se estableció incluir el material del cableado, accesorios y ductos. La información resultante del diseño de CCTV, acceso, asistencia y redes compone el insumo principal para este subsistema.

Abstracción operativa. El sistema requiere mantenimiento mínimo. Dependió del área de tecnología.

\section{Diseño de la arquitectura}

En la evaluación de los sistemas de forma integral se sugirió agregar un rack de telecomunicaciones en la oficina gerencia de sala, al centro del supermercado. Esto resultó en la optimización del cableado y mejor distribución de cargas de red. Se consideraron 3 puntos de distribución en total para el equipamiento del supermercado.

Con el relevamiento de información de cada especialidad se definió el requerimiento de equipos, características mínimas y servicios necesarios, se obtuvieron entonces los siguientes resultados. 


\section{CCTV, Acceso y Asistencia (Sistema integral)}

Dimensionamiento de componentes. De acuerdo con los requerimientos relevados en la etapa anterior y los planos actualizados del edificio se procedió al diseño de CCTV, acceso y asistencia como un sistema integral.
Para CCTV se consideraron necesarias funciones de monitoreo en tiempo real, grabación y búsqueda de vídeo, copia de seguridad de archivos, visualización en videowall. En este sentido, la solución CCTV comprende el equipamiento descrito en la tabla 1.

Tabla 1. Equipamiento para solución CCTV

\begin{tabular}{lll}
\hline Equipo & Cantidad & Descripción \\
\hline Grabadores & 2 & NVR de 16TB \\
\hline & 1 & Panorámica $180^{\circ}$ \\
& 1 & PTZ \\
& 2 & LPR \\
Cámaras & 18 & Domo \\
& 12 & Box \\
& 5 & Fish Eye \\
& 30 & Bala \\
\hline
\end{tabular}

La tabla 1 presenta el resumen de la revisión final de los especialistas y personal clave del supermercado. Se definen los tipos y cantidad por propósito con un total de 74 cámaras distribuidas en áreas de almacén, cajas, delivery, ingreso principal, ingreso de servicios, oficinas, parqueo, perímetro, plaza comercial y sala principal. Los tipos de cámaras de acuerdo con el área que monitorean son: bala y domo para vistas puntuales; lente variable y alta resolución para operaciones de caja; fisheye para amplia visualización de sala; reconocimiento de placas en delivery y el área de servicios; $180^{\circ}$ para el parqueo frontal; PTZ (pan, tilt, zoom) para una visualización de zonas a demanda en sala. Finalmente, con la cantidad y calidad definidos se dimensionaron 2 grabadores con capacidades de redundancia en almacenamiento, energía y conectividad y 1 equipo de monitoreo robusto para la visualización de varios hilos de video al mismo tiempo con 2 pantallas de operación 24/7. Dos de las cámaras demandan más de la capacidad PoE $+(30 \mathrm{w})$, se consideran fuentes dedicadas para estas cámaras.

El control de acceso requiere proteger y controlar el acceso para 6 puertas en todo el predio: 1 en oficinas, 1 en almacenes, 1 en tecnología, 1 gerencia de sala, 1 en sala de servidores y 1 en cuarto de comunicaciones. En esta etapa se seleccionaron los componentes de acceso de acuerdo con la proximidad de las puertas a cada sala de equipamiento donde se definió el lugar para las controladoras. Con las distancias en consideración, se seleccionaron las controladoras y se definió el cableado necesario. Todos los seguros se consideraron electromagnéticos de acuerdo con el tipo de apertura de puerta. La solución de control de acceso comprende el siguiente equipamiento: 4 paneles de control, 10 lectores de tarjeta, 2 botones de salida, 6 seguros electromagnéticos $y$ 
soportes por tipo de puerta, 100 tarjetas y 1 emisor de tarjeta.

Finalmente, para el control de asistencia se definieron dos puntos de control, uno en cada ingreso de los predios con soporte para lectura biométrica de huella dactilar y lector de tarjetas.

Alcance de servicios. Los servicios que fueron considerados para este sistema integral implicaron para CCTV: la instalación y configuración del equipamiento activo, montaje y alienación de cámaras, configuración de los grabadores y definición de reglas de comportamiento; Para acceso el montaje y configuración de paneles, instalación de accesorios de puerta; finalmente, asistencia requiere el montaje y configuración. Se considera la configuración de los 3 componentes a una sola consola de gestión central. También se consideraron las horas de capacitación al personal de tecnología y administrativo para la gestión de los 3 sistemas.

Conexiones de red y seguridad. Se consideraron las cargas estimadas de tráfico de datos hacia los grabadores, la carga de energía (PoE) de cámaras y la cantidad de puertos de CCTV. Asimismo, se contemplaron los puntos de red para el equipamiento de monitoreo y grabadores.

Para cada controladora de acceso se consideró un puerto de red en los 3 sitios de distribución. Los puntos para control de asistencia se contemplaron en cada ingreso al edificio: en la puerta de oficinas sur y en el acceso de servicios en la parte norte.
Se consideraron los puntos de red requeridos para el equipamiento de CCTV, acceso y asistencia para ser contemplados en el sistema de red de datos. Adicionalmente, el requerimiento de consumo eléctrico de las cámaras PoE.

Como diseño lógico se recomendó el uso de VLANs por propósito para aislar el tráfico entre los sistemas.

Integración de componentes. $\mathrm{El}$ diseño de estos 3 sistemas incluyó la demanda de puntos de red y consumo eléctrico para el sistema de red.

\section{Redes de datos y Seguridad}

\section{Dimensionamiento de componentes.}

En el diseño se consideraron todos los puntos de red, la carga PoE y el ancho de banda requeridos por los otros sistemas. Para el backbone de red se consideraron puertos de fibra con capacidad de crecimiento a 10Gbps. Considerando las distancias al equipamiento, en este paso se sugiere la inclusión de un tercer rack de distribución en medio de la sala para reducir costos de distribución.

De acuerdo con el diseño realizado el equipamiento mínimo necesario para la solución de redes es: 1 unidad Core (Capa 2 y 3), 3 unidades de acceso con 48 puertos (Capa 2), 3 unidades de acceso con 48 puertos PoE+ (Capa 2) y 4 transceivers de corto alcance. Se consideró el esquema para conexiones entre core y acceso presentado en el gráfico 2 . 


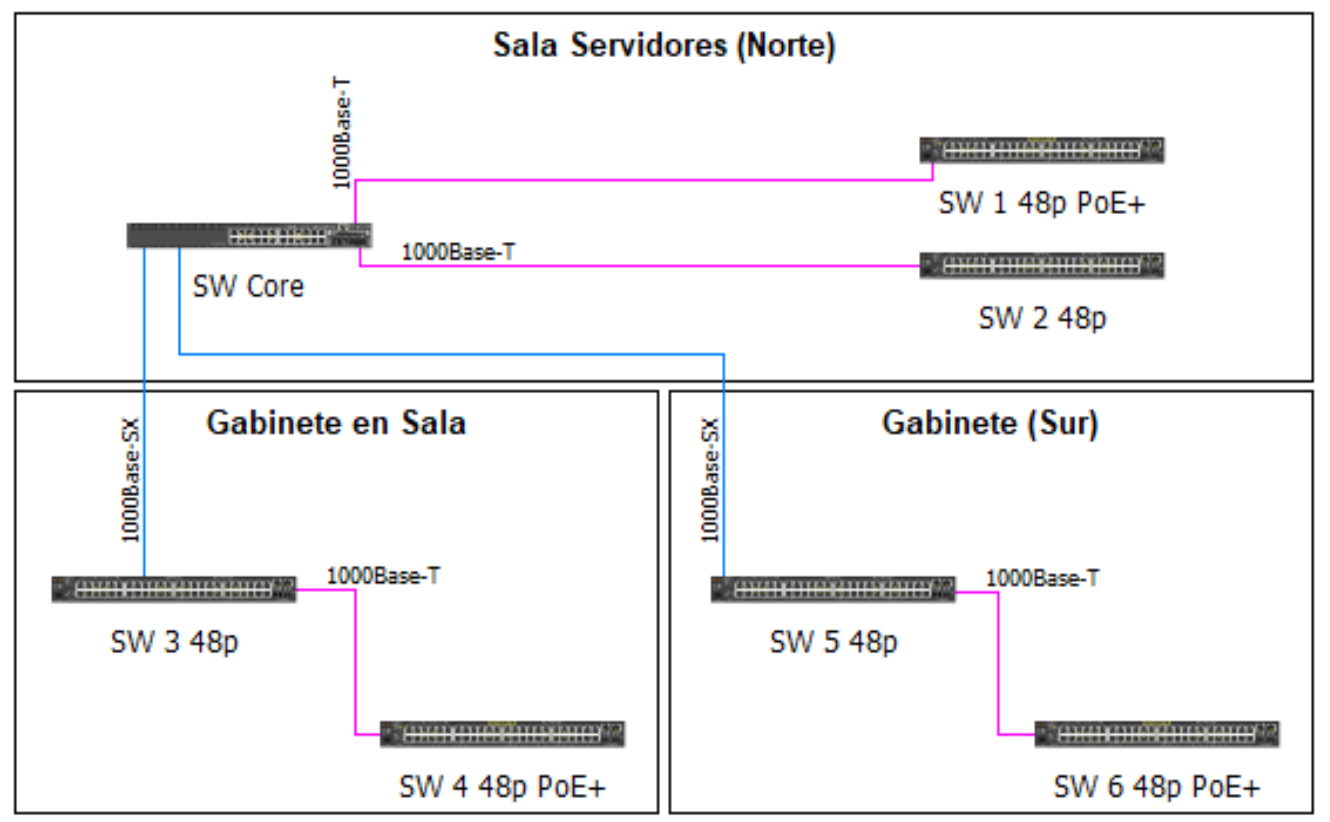

Gráfico 2. Dispositivos de red y conexiones

Según se aprecia en el gráfico 2, se contempló el core en sala de servidores. Este concentra las conexiones de los switches de acceso y brinda conectividad a los dispositivos conectados directamente (servidores, grabadores, firewall, etc.). La transmisión entre el core y switches de acceso principales es a través de fibra óptica a 1Gbps (líneas azules). La transmisión a switches dependientes del core y secundarios en cascada se realiza por cobre (líneas rosadas). En cada ubicación se cuenta con 1 switch de 48 puertos normal y 1 switch de 48 puertos PoE para acceso a dispositivos.

Asimismo, en la tabla 2 se presenta la composición del backbone de red.

Tabla 2. Composición del backbone

\begin{tabular}{llll}
\hline Origen & Velocidad - Medio & Destino & \\
\hline Core & 1 Gbps - Fibra & Gabinete Sala & SW \#3 \\
Core & 1 Gbps - Fibra & Gabinete Sur & SW \#5 \\
Core & 1 Gbps - Cobre & Servidores & SW \#1 \\
Core & 1 Gbps - Cobre & Servidores & SW \#2 \\
\hline
\end{tabular}

La tabla 2 muestra la composición de las conexiones del switch core hacia los switches de acceso. Para las conexiones de fibra se contemplaron transceivers de $1 \mathrm{Gbps}$ en cada extremo, éstos pudiendo ser reemplazados más adelante por módulos de 10Gbps utilizando la misma fibra instalada. 
Alcance de servicios. Los servicios de este apartado implican la instalación y configuración del equipamiento activo, configuración básica y diseño lógico de redes. También se consideró la capacitación al personal administrativo para el manejo de la red de datos.

Conexiones de red y seguridad. Se consideró el switch core y un firewall para concentrar todas las conexiones y aplicar filtros respectivamente. Se sugirió realizar el filtrado de la red entre redes internas por propósito y redes externas hacia internet $u$ otros destinos. El filtrado web para los usuarios es parte de los servicios del firewall.

Integración de componentes. Con el alcance descrito se contempló toda la parte activa de acceso a la red para todos los dispositivos mencionados en el alcance del proyecto. Asimismo, se habilitó la infraestructura base para brindar seguridad entre las redes internas y hacia la red externa del supermercado.

\section{Cableado estructurado}

Dimensionamiento de componentes. Considerando que la construcción se encontraba en una etapa de remodelación previa a la obra fina, se pudo diseñar mejores rutas y más estéticas para el cableado sin impactar en costos adicionales por empotrado de ductos o ductos sobrepuestos. Con la inclusión del punto medio de distribución, se mejoraron las rutas para el tendido de ductos y cableado. La estimación de cableado para CCTV asumió holgura adicional para mover las cámaras en la etapa de la instalación y así considerar que en la obra final del supermercado se presentarán luminarias o banners que pueden afectar la visibilidad. Se incorporó un soporte versátil para poder tener una altura variable en el montaje de las cámaras.

En la tabla 3 se muestra la distribución de racks y puntos de red contemplados para el proyecto.

Tabla 3. Puntos de red y accesorios

\begin{tabular}{ccccccccc}
\hline RACK & UBICACIÓN & TAMAÑO & DATOS & VOZ CAMARAS & $\begin{array}{c}\text { TOTAL } \\
\text { PUNTOS }\end{array}$ & PANELES & ORGANIZAdOR \\
\hline 1 & Sala & $16 U$ & 38 & 1 & 30 & $\mathbf{6 9}$ & 4 & 4 \\
2 & Sur & $42 U$ & 43 & 35 & 13 & $\mathbf{9 1}$ & 5 & 5 \\
3 & Servidores & $42 U$ & 42 & 34 & 31 & $\mathbf{1 0 7}$ & 6 & 6 \\
\hline TOTAL & & & 123 & 70 & 74 & $\mathbf{2 6 7}$ & $\mathbf{1 5}$ & $\mathbf{1 5}$ \\
\hline
\end{tabular}

La tabla 3 describe la cantidad de puntos de red contabilizados en todo el relevamiento y sus accesorios de conectividad y organización. La distribución incluye: puntos de datos para dispositivos y usuarios, puntos para telefonía y puntos de cámaras (éstos son energizados por los switches PoE, a excepción de dos unidades que requieren una fuente de energía adicional). 
Alcance de servicios. En el alcance de cableado estructurado se incluyó el montaje de racks, ductos y cableado con la certificación de todos los puntos de red para garantizar el funcionamiento.

Conexiones de red y seguridad. La infraestructura pasiva de red contempla el tendido del backbone de fibra entre racks y las conexiones entre switches y dispositivos en ambos extremos. Cada rack cuenta con una llave física codificada para personal autorizado.

Integración de componentes. No aplica.

\section{Consenso}

El consenso fue alcanzado en la $7 \mathrm{ma}$ iteración del proceso de diseño, cada iteración tomó en cuenta cambios en los requerimientos y aplicación restricciones presupuestarias y temporales.

Los trabajos para la instalación de equipamiento pasivo fueron coordinados en conjunto con obras civiles para ejecución durante la construcción del supermercado. En esta coordinación se tuvieron oportunidades de mejora para nuevas rutas de cableado e instalación de equipos con un impacto económico ventajoso para el supermercado. Por otro lado, algunos cambios de sistemas de climatización afectaron en tiempo al sistema de cableado estructurado. A continuación, se exponen algunas aclaraciones específicas para CCTV, acceso y cableado.

CCTV. La configuración del comportamiento de grabación y alertas en áreas de interés se realizó durante la ejecución de servicio. Asimismo, la configuración de los cuadros por segundo almacenados de acuerdo con la zona de monitoreo. La configuración de privilegios de acceso se define junto con el personal a cargo de la videovigilancia, supervisores y administradores. Se siguen los mismos lineamientos globales de ciberseguridad.

Acceso. La configuración e integración de control de acceso y asistencia se realizan a través de la misma consola de administración.

La configuración de privilegios de acceso se define junto con el personal a cargo de la videovigilancia, supervisores y administradores. Se siguen los mismos lineamientos globales de ciberseguridad. Se realiza la capacitación a sistemas como soporte del sistema y a recursos humanos para la alta y baja de credenciales. Se definen credenciales con diferentes niveles de acceso por usuario

Cableado. La coordinación de la ejecución del cableado estructurado con obras civiles fue de importancia significativa. En esta etapa de consenso se logró coordinar finamente los alcances de trabajo de cada participante. De esta manera, se lograron reducciones de costos en el tendido de ductos para el cableado estructurado.

\section{CONCLUSIONES}

Con rigor científico, con base en la literatura existente $y$ el conocimiento práctico, se propuso un proceso científico para el diseño de soluciones tecnológicas que integra 5 sistemas de redes de datos, seguridad, CCTV, control de acceso y cableado estructurado. Este proceso es iterativo e implica seguir cuatro etapas (generalidades, relevamiento de variables, diseño de arquitectura, consenso), cada etapa contempla a su vez condiciones necesarias para su aplicación. Se resaltó su utilidad dado que facilitará la 
implementación de soluciones tecnológicas integradas, propiciando la consecución de mejores resultados. En este sentido, se entiende que aplicar un procedimiento científicamente elaborado puede optimizar el uso de recurso generando ahorros considerables en tiempo, recurso humano y tecnología.

El proceso fue aplicado en un supermercado que desarrollaba actividades en La Paz, Bolivia, para este caso fueron necesarias 7 iteraciones en las que la participación de los especialistas de seguridad física, redes y cableado fue liderada por un arquitecto de soluciones tecnológicas. La aplicación de las etapas y consideraciones requeridas por el proceso fueron ajustando el diseño para cumplir con los requisitos, restricciones y expectativas del supermercado, generando así valor. Puesto que el proceso científico propuesto abstrae los requisitos del cliente y las capacidades tecnológicas del equipamiento actual para resolver las necesidades del cliente, es factible replicar su aplicación para otros escenarios en distintos países. El proceso es perfectible ante futuros adelantos tecnológicos.

\section{REFERENCIAS}

Allred, M. (2018). Aruba Certified Design Professional (HPE6-A47). HPE Press

Allied Telesis (2020). Enterprise Networking Solutions, solutions guide. Network Smarter. https://www.alliedtelesis.com/sites/de fault/files/documents/solutionsguides/ati-enterprise-networkingsolutions-sg.pdf

Cadenas, X., Zaballos, A. y Salas S. (2012). Guía de sistemas de Cableado Estructurado. Ediciones Experiencia

Cano-Pita, G. E. (2018). Las TICs en las empresas: evolución de la tecnología y cambio estructural en las organizaciones. Dominio de las Ciencias, 4(1), 499-510.

Dallabona, L. F., Nardelli, L. T., y Fernandes, A. R. V. (2019). Variáveis Contingenciais e Sistemas de Controle Gerencial predominantes em uma rede de supermercados do Brasil. Revista Evidenciação Contábil \& Finanças, 7(1), 58-77

Migga, J. (2017). Guide to Computer Network Security. Springer

Ministerio del Trabajo (2015). Resolución Ministerial No. 153/15. Bolivia

Sennewald, C (2012). Security Consulting. Elsevier 